\title{
Gallium Nitride as Transparent Electron-Selective Contact in Silicon Heterojunction Solar Cells
}

\author{
Angela N. Fioretti, Tzu-Chin Chang Chien, Yashi Xiao, Christophe Ballif, and Mathieu Boccard \\ Photovoltaics and Thin Film Electronics Laboratory, Institute of Microengineering, Ecole Polytechnique \\ Fédérale de Lausanne (EPFL), Rue de la Maladière 71b, CH-2000 Neuchâtel, Switzerland
}

\begin{abstract}
Carrier-selective, passivating contacts have allowed silicon heterojunction (SHJ) cells to reach recordbreaking efficiencies particularly in all-back-contacted designs. However, two-side-contacted SHJ cell efficiency has been limited due in part to parasitic absorption losses up to $3 \mathrm{~mA} / \mathrm{cm}^{2}$ in the a$\mathrm{Si}$ :H layers. More transparent materials could reduce this current loss while minimizing process complexity. Gallium nitride (GaN), with a bandgap of $3.4 \mathrm{eV}$ and an advantageous band alignment with silicon, could be applied as a transparent electron-selective layer. Here, we report on SHJ solar cells utilizing PECVD GaN layers grown at $200^{\circ} \mathrm{C}$ as electron-selective contact. First devices exhibited open-circuit voltages of $\sim 575 \mathrm{mV}$ due to poor passivation, and low conductivity of the as-yet undoped GaN layers induced high series resistance (Rs). However, this $V_{\text {oc }}$ suggests the potential for electron selectivity if appropriate passivation and doping strategies are implemented.
\end{abstract}

Index Terms - photovoltaic cells, heterojunctions, passivating contacts, nitrides.

\section{INTRODUCTION}

Passivating, carrier-selective contact materials for silicon heterojunction (SHJ) solar cells are increasingly gaining research attention due to the success of amorphous silicon and poly-silicon based contacting schemes [1]. Low transparency and/or poor thermal stability of the thin film silicon layers currently represent the largest drawbacks to these otherwise highly successful contact designs. In light of this, researchers have made significant strides in development of non-siliconbased contacting strategies, most notably in contacts utilizing transition metal oxides (TMOs) such as $\mathrm{TiO}_{\mathrm{x}}$ and $\mathrm{MoO}_{\mathrm{x}}$ [2], [3]. However, thermal stability and sensitivity to plasma exposure are persistent problems for transition metal oxide based contacts, thus introducing limitations to the solar cell fabrication process. Such challenges have encouraged researchers to look outside the TMO family for equally transparent yet more robust candidate materials for selective contact design.

Wide bandgap nitride materials are an attractive alternative to TMOs due to their ability to withstand high temperature processing and their long research history in optoelectronic applications. Gallium nitride in particular has a bandgap of $3.4 \mathrm{eV}$, giving it similar transparency to $\mathrm{TiO}_{\mathrm{x}}$ and $\mathrm{MoO}_{\mathrm{x}}$, and can be deposited by a variety of methods [4]. Importantly, $\mathrm{GaN}$ exhibits an advantageous band alignment with crystalline $\mathrm{Si}$, specifically a valence band offset of $\sim 2 \mathrm{eV}$ and a

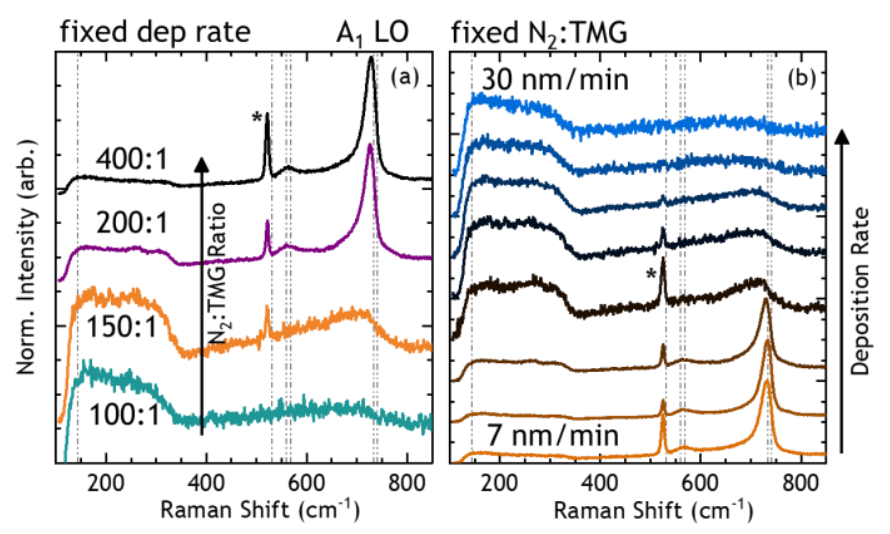

Fig. 1 (a) Raman of GaN films deposited on silicon with varying $\mathrm{N}_{2}$ :TMG flow rate ratio. The asterisk denotes the silicon substrate peak, and vertical grey lines give the Raman modes of wurtzite $\mathrm{GaN}$ [6]. A ratio of at least 200:1 was necessary to obtain the $\mathrm{GaN}$ phase. (b) Raman spectra taken from a representative $\mathrm{GaN}$ layer deposited on silicon with a thickness gradient spanning 25$100 \mathrm{~nm}$. Regions of the film with the fastest deposition rate (blue and black curves) appear amorphous by Raman, while regions with the slowest deposition rate (orange curves) exhibit GaN peaks.

conduction band offset of $\sim 0.5 \mathrm{eV}$, making it a good candidate for an electron-selective layer if the appropriate work function can be achieved [5]. The demonstrated ability to grow $\mathrm{GaN}$ at $<400{ }^{\circ} \mathrm{C}$ [6], in addition to well-documented n-type doping strategies, further supports the suitability of this material for application in carrier selective contacts.

In this contribution, we present $\mathrm{GaN}$ prepared at $200{ }^{\circ} \mathrm{C}$ using plasma-enhanced chemical vapor deposition (PECVD). Layers were grown directly on textured silicon wafers as the transparent back contact material, and Raman spectroscopy was used to identify the GaN phase. Current-voltage (JV), external quantum efficiency (EQE), and variable-illumination $\mathrm{JV}$ were used to assess the contact performance. It was found that cells with open-circuit voltage of $\sim 575 \mathrm{mV}$ could be achieved with these GaN layers, but that high resistivity in the nitride film led to recombination losses at the back contact. These findings direct future research efforts on $\mathrm{GaN}$ integration in SHJ cells toward implementation of effective doping and passivation strategies.

\section{METHODS}

A. Solar Cell Fabrication and Characterization 


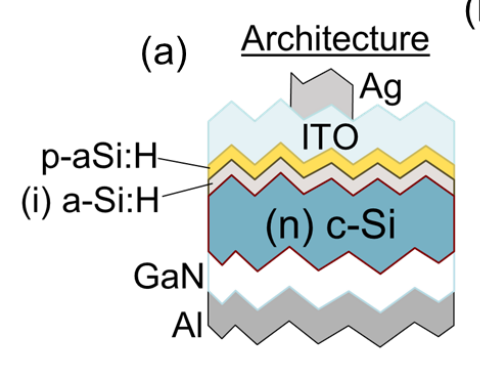

(b) Post-Anneal

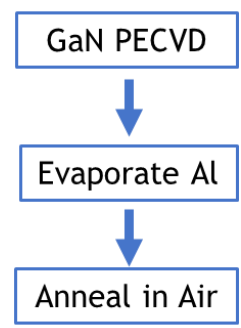

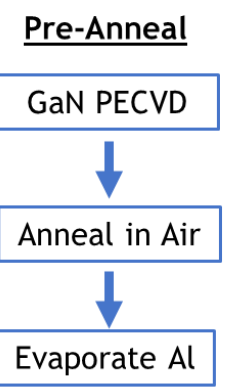

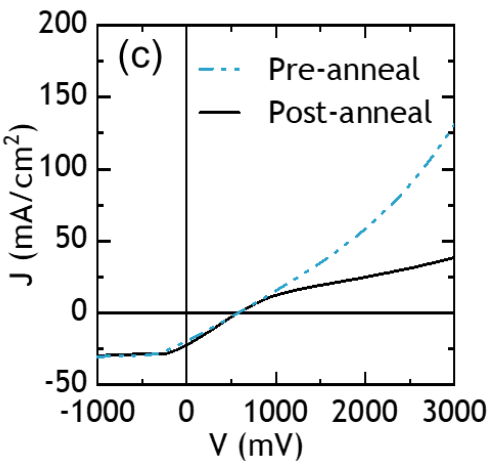

Fig. 2 (a) Schematic depicting the SHJ solar cell architecture used to test the performance of electron-selective contacts fabricated with PECVD GaN films. (b) Processing steps for fabricating the back contact, showing either post-annealing (i.e. after Al evaporation) or preannealing (before Al evaporation). (c) Representative JV curves for pre-annealed (blue dashed line) or post-annealed (black solid line) GaNcontacted cells, in which current saturation at high forward bias occurs if cells are annealed after Al evaporation but not if they are annealed before.

Solar cells were prepared on textured, 4", $n$-type float zone wafers with resistivity of $2-3 \Omega \mathrm{cm}$, and thickness of 180-220 $\mu \mathrm{m}$. Plasma-enhanced chemical vapor deposition (PECVD) was used to deposit front thin-film silicon layers to fabricate the $1 \times 1 \mathrm{~cm}^{2}$ standard all-silicon reference cell as described elsewhere [7]. For back GaN layers, PECVD conditions were as follows: tri-methyl gallium (TMG), $\mathrm{N}_{2}$, and $\mathrm{H}_{2}$ were used as precursor gases in a $110 \mathrm{MHz}$ plasma held at $30 \mathrm{~W}$ power for all depositions. Chamber temperature during deposition was $200{ }^{\circ} \mathrm{C}$ and films were deposited to a thickness of $40 \mathrm{~nm}$ as calibrated by co-deposited films on glass measured with stylus profilometry. For the different back surface pretreatments investigated in Fig. 3, amorphous silicon layers were first deposited on the front side of the wafer, and then wafers were left as-is ("oxide"), dipped in $1 \% \mathrm{HF}$ for 1 min to remove the native oxide ("HF-dip"), or dipped in HF and then exposed to $1: 1 \quad \mathrm{~N}_{2}: \mathrm{H}_{2}$ plasma for 10 minutes ("N2/H2") immediately prior to GaN deposition. Front-side tin-doped indium oxide (ITO) and silver were deposited by sputtering through separate $1 \times 1 \mathrm{~cm}^{2}$ shadow masks to define the cell area and metal grid (three cells per wafer, also for the all-silicon standard cell). Aluminum full-area back metallization was evaporated at room temperature to a thickness of approximately $100 \mathrm{~nm}$. Wafers were annealed at $210^{\circ} \mathrm{C}$ for 20 minutes in air either before or after aluminum evaporation, as described in Fig. 2.

Current-voltage characteristics (JV) were collected on finished devices using a Wacom Electric Co. Super Solar Simulator with AM $1.5 \mathrm{G}$ illumination and Keithley sourcemeters. For series resistance extraction, a variableillumination method was carried out as described in a previous publication [8]. External quantum efficiency (EQE) was collected using a home-built spectral response measurement system, equipped with xenon arc lamp.

\section{B. Material Characterization}

Raman spectroscopy was carried out using a $325 \mathrm{~nm} \mathrm{He}-\mathrm{Cd}$ laser in a MonoVista CRS+ Raman spectrometer equipped with VistaControl software. The measurements were collected at room temperature, with a collection time of $10 \mathrm{sec}$ accumulated over six repeated measurements. Samples for Raman spectroscopy were deposited as described above on polished (100) Si substrates with the native oxide intact, and were deposited to a thickness of $70 \mathrm{~nm}$ (in the case of fixed deposition rate) or between $35-150 \mathrm{~nm}$ (in the case of variable deposition rate). Variable deposition rate films were obtained by utilizing unintentional inhomogeneity produced inside the chamber during GaN deposition. Substrates large enough to span the inhomogeneous region were manually masked with marker lines to define multiple regions of different thickness. Marker lines were removed with acetone lift-off after deposition. Each region was then measured for thickness using stylus profilometry, and the thicknesses were divided by the deposition time, which was the same for the different regions, to determine the variable deposition rates.

\section{RESULTS AND DISCUSSION}

Fig. 1 displays Raman spectra collected from PECVDdeposited GaN films on (100) silicon deposited at $200{ }^{\circ} \mathrm{C}$. Characteristic Raman modes for bulk wurtzite $\mathrm{GaN}$ are indicated by solid vertical grey lines [6], and the characteristic Raman mode for crystalline silicon is indicated by an asterisk in each sub-figure. Fig. 1a displays Raman spectra taken of a set of GaN films deposited with increasing $\mathrm{N}_{2}$ flow rate, while the tri-methyl gallium (TMG) flow rate was kept constant. It was found that a flow rate ratio of at least 200:1 $\mathrm{N}_{2}$ :TMG was needed to achieve a crystalline GaN film, as determined by the appearance of the $\mathrm{A}_{1}$ longitudinal optic $\left(\mathrm{A}_{1} \mathrm{LO}\right)$ Raman mode at approximately $719 \mathrm{~cm}^{-1}$ for this flow rate ratio. In both the 200:1 and 400:1 case, the $A_{1}$ LO peak is shifted to lower values compared to the bulk peak position $\left(734 \mathrm{~cm}^{-1}\right)$, indicating nanocrystalline material [6]. In Fig. 1b, multiple spectra taken at different regions of the same thickness gradient film are 

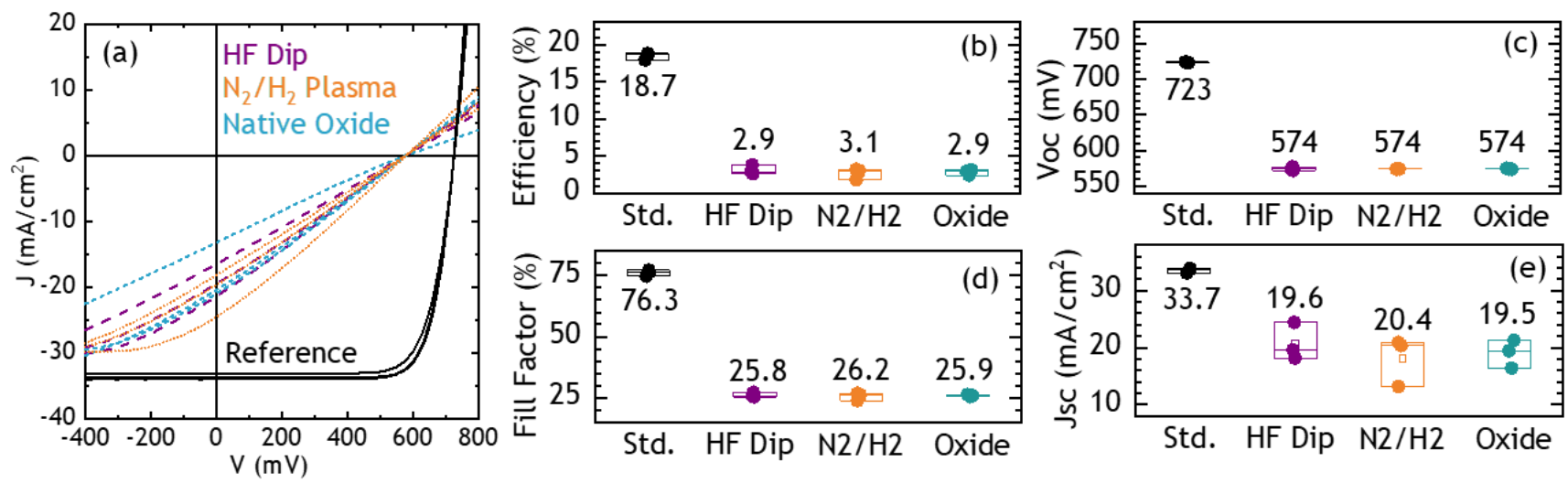

Fig. 3 (a) JV curves of GaN-contacted cells in which the back silicon surface was treated in one of three ways. The reference cells shown in black were prepared in the same way, but with n-aSi:H/ITO/Ag contacts at the back. Ignoring random variation from cell to cell on each wafer, the back surface pretreatments did not significantly change the overall JV performance. Instead, all cells were dominated by high series resistance, very likely due to the insulating character of undoped GaN. (b)-(e) Figures of merit for each set of three cells as well as for the all-silicon reference ("Std."). Numbers noted in black give the average value for each condition. Notably, the $\mathrm{V}_{\mathrm{oc}}$ for GaN-based cells was able to reach $574 \mathrm{mV}$, suggesting that $\mathrm{GaN}$ provides some electron selection even though the cells suffer from poor passivation.

displayed, with the thickest region $(\sim 150 \mathrm{~nm})$ in blue at the top and the thinnest region $(\sim 35 \mathrm{~nm})$ in orange at the bottom. Film regions with the highest deposition rate showed no Raman peaks, indicating amorphous material, while film regions with the slowest deposition rate $(\sim 7 \mathrm{~nm} / \mathrm{min})$ again exhibited the $\mathrm{A}_{1} \mathrm{LO}$ mode. Generally, these results highlight two important points for deposition GaN films by PECVD: first, a high $\mathrm{N}_{2}$ over-pressure is required to provide enough active nitrogen to the chemical reaction, and second, a slow deposition rate is required to achieve crystalline films rather than amorphous.

Fig. 2a depicts the cell architecture used to test the selective contact performance of 40-nm-thick PECVD GaN layers deposited with a 400:1 flow rate ratio of $\mathrm{N}_{2}$ :TMG. Although the ultimate goal is for GaN to be combined with a TCO and metal grid either at the front of the cell in a rear junction device or at the back in the case of a bifacial solar cell, a fullarea $\mathrm{Al}$ contact is used here for this initial investigation, which is a common approach for initial investigations of novel electron-contact layers [7], [9]. Fig. 2b demonstrates the two alternative processes tested to determine the best timing for annealing the cells after sputtering the front contact (a necessary step to ensure recovery of front passivation after sputter-induced damage). In the first case, cells were annealed after aluminum evaporation, and in the second case, cells were annealed after $\mathrm{GaN}$ deposition but before aluminum evaporation. Fig 2c shows representative JV curves for cells that experienced pre-annealing (blue dashed line) compared to cells that experienced post-annealing (purple solid line). In the case of post-annealing, current begins to saturate at high forward bias (above about $800 \mathrm{mV}$ ), contributing to a large Sshape in the JV curve. In the case of pre-annealing, the same effect is not observed, although a high ohmic resistance can be seen. The cause for this difference is unclear, but could be due to passivating interface defects between the $\mathrm{GaN}$ and the $\mathrm{Al}$ as a result of annealing the GaN layer in air, or alternatively could be related to minimizing detrimental reactions occurring between the $\mathrm{Al}$ and $\mathrm{GaN}$ layers upon post-annealing. Nonetheless, all remaining cells presented in this work were fabricated using the pre-annealing strategy to avoid the current-saturation effect.

Fig. 3 shows current-voltage (JV) characteristics for GaNcontacted cells that each received one of three back-surface pretreatments before deposition of the GaN layer. JV characteristics for a set of three all-silicon standard cells prepared in a similar way but including n-aSi:H/ITO/Ag at the back contact are also shown for reference. In the first pretreatment case, wafers were subjected to a typical HF dip immediately prior to loading into the PECVD chamber, thus exposing bare silicon dangling bonds to the GaN deposition atmosphere. In the second case, wafers were subjected to the same HF dip, immediately loaded into the PECVD chamber, and then exposed to 10 min of $\mathrm{N}_{2}+\mathrm{H}_{2}$ plasma prior to $\mathrm{GaN}$ deposition. In the third case, the native oxide on the wafer was left intact, and the GaN film was deposited directly on top. These three surface pretreatments were investigated to assess whether the nitrogen plasma exposure during GaN deposition could provide any surface passivation at the back contact (e.g. via formation of a $\mathrm{SiN}_{\mathrm{x}}$ layer), or if this exposure had no effect compared to the untreated surface with the native oxide.

Generally, the back surface exposure to $\mathrm{N}_{2}$ plasma had no effect on the overall cell performance of the GaN-based cells, nor did it contribute to an increase in $\mathrm{V}_{\mathrm{oc}}$ (as would be expected for improved passivation). Instead, all cells with GaN back contacts exhibited significant series resistance (Fig. 3a), which led to losses in fill factor (FF), efficiency, and even short-circuit current $\left(\mathrm{J}_{\mathrm{sc}}\right)$. Such high ohmic resistance is very likely caused by the insulating nature of undoped GaN. The $\mathrm{V}_{\text {oc }}$ of the GaN-based cells was consistent across all three surface pretreatments at about $575 \mathrm{mV}$. Considering the lack of a passivation layer, this $\mathrm{V}_{\text {oc }}$ suggests the GaN layers are 


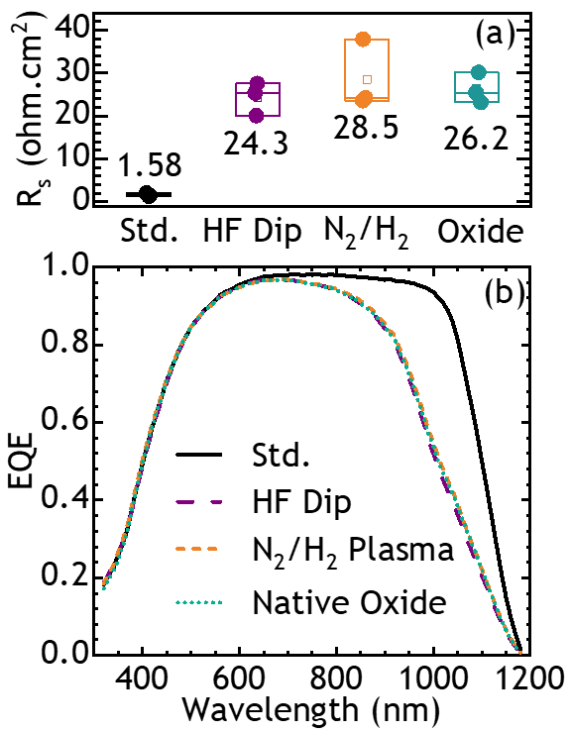

Fig. 4 (a) Series resistance $\left(\mathrm{R}_{\mathrm{s}}\right)$ for the all-Si standard cell compared to that of the GaN-contacted cells, as determined from variable-illumination JV. Whereas the standard cell exhibits $R_{s}$ of about $1.6 \mathrm{ohm} . \mathrm{cm}^{2}, \mathrm{R}_{\mathrm{s}}$ of $\mathrm{GaN}$-contacted cells is much higher: approximately $25 \mathrm{ohm} . \mathrm{cm}^{2}$. (b) Representative EQE curves for GaN-contacted cells with each pretreatment compared to the standard cell. Large losses are observed in the range from 800$1200 \mathrm{~nm}$, indicating significant back surface recombination.

providing some level of carrier selectivity, which might be enhanced by modulating the GaN work function via n-type doping. However, such $\mathrm{V}_{\text {oc }}$ could also be set by the wafer doping, indicating in this case that the GaN layer acts only as a spectator in the contact stack. Further investigation is necessary in order to determine whether the band offsets of $\mathrm{GaN}$ with c-Si are enough on their own to provide selective electron transport.

Fig. 4a presents the series resistance $\left(\mathrm{R}_{\mathrm{s}}\right)$ extracted from variable-illumination JV measurements for the all-silicon standard cell compared to the GaN-contacted cells. As expected from the JV curves, the GaN-based cells exhibit extremely high series resistance around $25 \Omega \mathrm{cm}^{2}$, with little effect of the different back surface pretreatments. Ultimately, it is this high resistance that obscures any detailed comparison of these cells. Fig. $4 \mathrm{~b}$ presents external quantum efficiency (EQE) measurements of representative cells from each category shown in Fig. 3. The three GaN-contacted cells exhibit strong losses compared to the reference cell in the long wavelength range $(800-1200 \mathrm{~nm})$, as well as some smaller losses in the range $600-800 \mathrm{~nm}$. In the range $>900 \mathrm{~nm}$, the observed reductions in EQE originate partly from poor internal reflectance from the $\mathrm{Al}$ reflector near the back contact interface [10]. However, the dominant effect leading to reductions in the $\mathrm{EQE}$ is likely high interface recombination at the back contact and can be understood as arising from a lack of effective passivation at the interface with the silicon wafer. Again, exposure to the $\mathrm{N}_{2}$ plasma during $\mathrm{GaN}$ deposition did
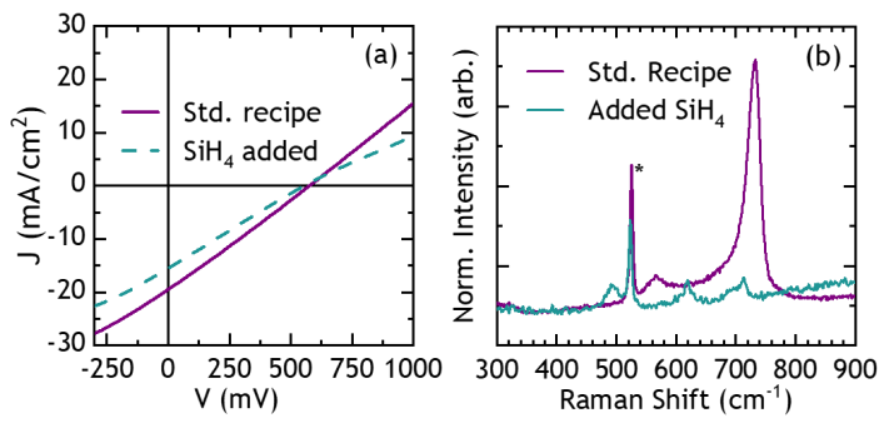

Fig. 5 (a) Representative JV curve for a GaN-contacted cell in which the nitride layer was deposited with added $\mathrm{SiH}_{4}$ (blue dashed line) compared to cell made with the standard $\mathrm{GaN}$ recipe (purple solid line). No improvement to series resistance was observed, but an S-shape at $\mathrm{V}_{\mathrm{oc}}$ was introduced. (b) Raman spectroscopy of the $\mathrm{SiH}_{4}$-containing $\mathrm{GaN}$ compared to the standard $\mathrm{GaN}$ recipe, in which a significant loss in film crystallinity occurred, in addition to observing amorphous silicon (shoulder near the substrate peak just below $500 \mathrm{~cm}^{-1}$ ). The broad mode near $620 \mathrm{~cm}^{-1}$ may result from a $\mathrm{SiN}_{\mathrm{x}}$ secondary phase.

not improve back surface recombination compared to the bare silicon or native oxide case, which is consistent with the similar JV performance and $\mathrm{V}_{\text {oc }}$ observed for all GaN-based cells in Fig. 3.

Fig. 5 displays the results of a preliminary doping attempt utilizing added $\mathrm{SiH}_{4}$ as the source of n-type dopant. Given that $\mathrm{Si}$ is a well-known donor dopant in high-temperature $\mathrm{GaN}$ growth, it was expected to incorporate as an n-type dopant in the low-temperature PECVD process as well. However, as observed in the representative JV curves in Fig. 5a, adding $\mathrm{SiH}_{4}$ to the deposition environment does not improve series resistance, but instead introduces a kink in the JV curve at $\mathrm{V}_{\mathrm{oc}}$ (blue dashed line). When examining the GaN layer more closely with Raman spectroscopy (Fig. 5b), it was found that adding $\mathrm{SiH}_{4}$ to the gas mixture resulted in a loss of $\mathrm{GaN}$ crystallinity and the appearance of an amorphous silicon phase (shoulder to the left of the substrate peak, marked with an asterisk). Overall, this phase separation indicates that the ratio between $\mathrm{SiH}_{4}$ and $\mathrm{TMG}$ was too high to be in the doping regime. Further experiments to develop effective n-type doping strategies in PECVD GaN are underway.

\section{CONCLUSIONS}

PECVD-deposited GaN layers were applied as transparent electron-selective contact in 1 x $1 \mathrm{~cm}^{2}$ SHJ solar cells. Although the layers were identified as the desired $\mathrm{GaN}$ phase by Raman spectroscopy, the layers imparted high series resistance and back contact recombination losses when integrated into SHJ cells. In order to test the effect of exposing the back silicon surface to $\mathrm{N}_{2}$ plasma during GaN PECVD, three pretreatment methods were investigated (HF dip, intentionaly $\mathrm{N}_{2}$ plasma exposure for $10 \mathrm{~min}$, or leaving the 
native oxide intact). It was found that no passivation effect was imparted to the contact stack by exposure to the $\mathrm{N}_{2}$-containing plasma, and in fact all cells exhibited a $\mathrm{V}_{\mathrm{oc}}$ of approximately $575 \mathrm{mV}$. Finally, n-type doping was attempted by introducing $\mathrm{SiH}_{4}$ to the precursor gas mixture during GaN deposition, but this resulted in formation of an amorphous silicon secondary phase and loss of GaN crystallinity. Overall, effective doping strategies must be developed if the benefits of reduced parasitic absorption from the wide gap GaN layer are to be fully realized.

\section{ACKNOWLEDGEMENTS}

This project has received funding from the European Union's Horizon 2020 Marie Skłodowska-Curie Actions under Grant Agreement No. 792720 (CLAReTE). Funding from the Swiss National Science Foundation (SNF) through the Ambizione Energy grant "ICONS" is also acknowledged. The authors would like to thank Christophe Allebe and Fabien Debrot from CSEM for high quality wet processing of the silicon wafers. Additional thanks to Vincent Paratte of EPFL for PECVD deposition of the amorphous silicon layers.

\section{REFERENCES}

[1] J. Melskens, B. W. H. van de Loo, B. Macco, L. E. Black, S. Smit, and W. M. M. Kessels, "Passivating Contacts for Crystalline Silicon Solar Cells: From Concepts and Materials to Prospects," IEEE J. Photovoltaics, vol. 8, no. 2, pp. 373-388, Mar. 2018.

[2] B. Liao, B. Hoex, A. G. Aberle, D. Chi, and C. S. Bhatia,
"Excellent c-Si surface passivation by low-temperature atomic layer deposited titanium oxide," Appl. Phys. Lett., vol. 104, no. 25, p. 253903, Jun. 2014.

[3] J. Geissbühler et al., "22.5\% Efficient Silicon Heterojunction Solar Cell With Molybdenum Oxide Hole Collector," Appl. Phys. Lett., vol. 107, no. 8, 2015.

[4] H. Amano, "Nobel Lecture: Growth of GaN on sapphire via low-temperature deposited buffer layer and realization of $p$ -type $\mathrm{GaN}$ by $\mathrm{Mg}$ doping followed by low-energy electron beam irradiation," Rev. Mod. Phys., vol. 87, no. 4, pp. 1133-1138, Oct. 2015.

[5] C. G. Van De Walle and J. Neugebauer, "Universal alignment of hydrogen levels in semiconductors, insulators and solutions," 2003.

[6] A. M. Jeffries et al., "Gallium nitride grown by molecular beam epitaxy at low temperatures," 2017.

[7] A. N. Fioretti, M. Boccard, A. C. Tamboli, A. Zakutayev, and C. Ballif, "Nitride layer screening as carrier-selective contacts for silicon heterojunction solar cells," in AIP Conference Proceedings, 2018, vol. 1999, no. 1, p. 040007.

[8] A. N. Fioretti, M. Boccard, R. Monnard, and C. Ballif, "Low Temperature p-type Microcrystalline Silicon as Carrier Selective Contact for Silicon Heterojunction Solar Cells," IEEE J. Photovoltaics, 2019.

[9] X. Yang et al., "Tantalum Nitride Electron-Selective Contact for Crystalline Silicon Solar Cells," Adv. Energy Mater., vol. 8, no. 20, p. 1800608, Jul. 2018.

[10] Z. C. Holman, S. De Wolf, and C. Ballif, "Improving metal reflectors by suppressing surface plasmon polaritons: a priori calculation of the internal reflectance of a solar cell," Light Sci. Appl., vol. 2, no. 10, pp. e106-e106, Oct. 2013. 